\title{
Impact of HIV Status, CD4 Count and Antiretroviral Treatment on Tuberculosis Treatment Outcomes in a Low-Burden Country
}

\author{
Vera Clérigo $^{1 *}$, Teresa Mourato², Conceição Gomes², Ana Castro² \\ ${ }^{1}$ Pulmonology Service-Thorax Department, North Lisbon Hospital Center, Lisbon, Portugal \\ ${ }^{2}$ Pulmonary Diagnostic Center Dr. Ribeiro Sanches, Lisbon, Portugal \\ Email: *vclerigo21@gmail.com
}

How to cite this paper: Clérigo, V., Mourato, T., Gomes, C. and Castro, A. (2018) Impact of HIV Status, CD4 Count and Antiretroviral Treatment on Tuberculosis Treatment Outcomes in a Low-Burden Country. Journal of Tuberculosis Research, 6, 281-291.

https://doi.org/10.4236/jtr.2018.64025

Received: September 27, 2018

Accepted: November 12, 2018

Published: November 15, 2018

Copyright $\odot 2018$ by authors and Scientific Research Publishing Inc. This work is licensed under the Creative Commons Attribution International License (CC BY 4.0).

http://creativecommons.org/licenses/by/4.0/

(c) $\underset{\mathrm{EY}}{\mathrm{C}}$ Open Access

\begin{abstract}
Background: Few data is available in low-burden TB-HIV countries on TB treatment outcomes. We evaluated TB patients (pts) characteristics by HIV status and compared treatment outcomes in relation to HIV status, CD4 count and antiretroviral therapy (ART). Methods: We performed a retrospective analysis of 447 adult TB pts who registered and initiated treatment in our center from 2014 to 2015. Results: Pts were categorized as HIV-negative ( $\mathrm{n}=$ 399, 89.3\%), HIV-positive on ART $(\mathrm{n}=42,9.4 \%)$ and HIV-positive not on ART $(\mathrm{n}=6,1.3 \%)$. The proportion of pts with extended TB therapy was higher among HIV-positive pts $(\mathrm{p}=0.03)$. Increased age was associated with higher death rates regarding treatment success (OR 1.08; 95\% CI 1.01-1.17, p = $0.03)$. Statistically significant higher mortality was found among HIV-positive pts on ART (OR 9.93; 95\% CI $1.36-72.37, \mathrm{p}=0.03$ ) and HIV-positive pts not on ART (OR 397.00; 95\% CI 44.27 to $3559.91, \mathrm{p} \leq 0.0001$ ) compared to HIV-negative pts. When multivariate analyses were restricted to HIV-positive pts, being not on ART was associated with higher mortality (OR 40.0; 95\% CI 4.37 - 365.78, $\mathrm{p}=0.001)$. Conclusions: There was significant difference in death rates between HIV-positive and HIV-negative TB pts. HIV-positive pts not on ART had a significantly higher mortality.
\end{abstract}

\section{Keywords}

Tuberculosis Treatment Outcomes, HIV Infection

\section{Introduction}

Tuberculosis (TB) is an international health problem, with an estimated 6.3 million new TB cases and 1.3 million deaths in 2016 [1], and has been acknowl- 
edged as one of the leading causes of death among human immunodeficiency virus (HIV)-infected patients [2]. In 2016, the majority of the estimated TB cases occurred in Asia (45\%) and the World Health Organization (WHO) African Region (25\%). Less significant number of TB cases happened in the Eastern Mediterranean Region (7\%), European Region (3\%) and Americas Region (3\%) [1]. In recent years, there has been a decline in the incidence of TB in Portugal, reaching the 20/100,000 population threshold in 2014. Additionally, there was also a decrease in the reported number of new HIV infection cases. The decline in TB incidence stands at 18/100,000 inhabitants in 2016 [3]. Compared to HIV-negative patients, HIV-positive TB patients can be more challenging to TB centers. TB-HIV co-infection poses more difficulties in the management of both diseases that may result in adherence decrease due to immune reconstitution inflammatory syndrome, high tablet problem with drug interactions and challenging toxicity profiles [4]. To reach the ideal approach, patients need standardized anti-tuberculosis treatment, while HIV-positive TB patients requisite additional antiretroviral treatment (ART). Furthermore, HIV-positive TB patients are more likely to be infectious for longer and to have diagnostic postponements due to the disease atypical presentation, resulting in poorer TB treatment outcomes [5] [6]. It is recognised that initiation of ART earlier in HIV infection improves outcomes, and international guidelines have been updated to reflect this. Data suggest that early TB treatment in HIV-infected people reduces morbidity and mortality, and appropriate treatment regimens are often successful [1].

HIV infection may severely influence TB treatment outcomes. Several studies with mixed results from high-burden TB countries have considered the impact of HIV infection on TB treatment outcomes. However, few data is available in low-burden TB-HIV countries. In high-burden TB/HIV settings, some studies have revealed worse TB treatment outcomes in HIV-positive TB patients when paralleled to HIV-negative TB patients [7]-[12], while other data established that TB treatment outcomes did not diverge between the two groups [13] [14]. Since the initiation of ART, it has been progressively recognized that HIV-positive TB patients are a heterogeneous group, and TB mortality during TB treatment will diverge between HIV-positive TB patients on ART and those not on ART. To date, few data are available in low-burden TB-HIV countries on TB mortality in HIV-positive TB patients on ART, and HIV-positive TB patients not on ART comparative to HIV-negative TB patients. In order to guarantee resources availability for TB and HIV management, it is imperative that countries monitor for co-infection in order to diagnose, treat and prevent TB-HIV co-infection, thus decreasing TB burden and increasing the quality of life of people living with HIV.

The aim of the study, therefore, was to evaluate TB patients' characteristics by HIV status and compare TB treatment outcomes (mortality and success) in relation to HIV status, CD4 count and ART in HIV-positive patients on ART, and 
HIV-positive patients not on ART compared to HIV-negative TB patients attending a Pulmonary Diagnostic Center in Lisbon, Portugal. Additionally, we aimed to study the impact of ART status and CD4 status on TB treatment outcomes among HIV-positive TB patients.

\section{Materials and Methods}

\subsection{Population and Design}

We performed a retrospective cohort study conducted at the Pulmonology Diagnosis Center Dr. Ribeiro Sanches that provides TB diagnosis, treatment and monitoring to the regional area of Lisbon-the capital city of Portugal. This center is one of the major public health centers in Portugal that provides service for people with TB. TB services are fully integrated in this center: medical and nursery consultations with periodic monitoring and evaluation, the adherence support and TB treatment dispensed by trained TB nurses.

\subsection{Inclusion and Exclusion Criteria}

We included all new adult TB patients (aged $\geq 18$ years) who registered and initiated TB treatment in our center between 1 January 2014 and 31 December 2015. We excluded 101 patients: 50 patients for whom treatment outcomes were inconclusive (unknown or transferred out), 44 patients who failed treatment and 7 patients who had not concluded treatment for unsupported diagnosis (Figure 1). All patients had known HIV status, according to the need to screen at least $80 \%$ of all TB patients for HIV as proposed by the WHO recommendations.

\subsection{Data Collection}

Data from the Center registry database were collected and completed by reviewing patient clinical records and paper-based TB registers. Variables for all patients

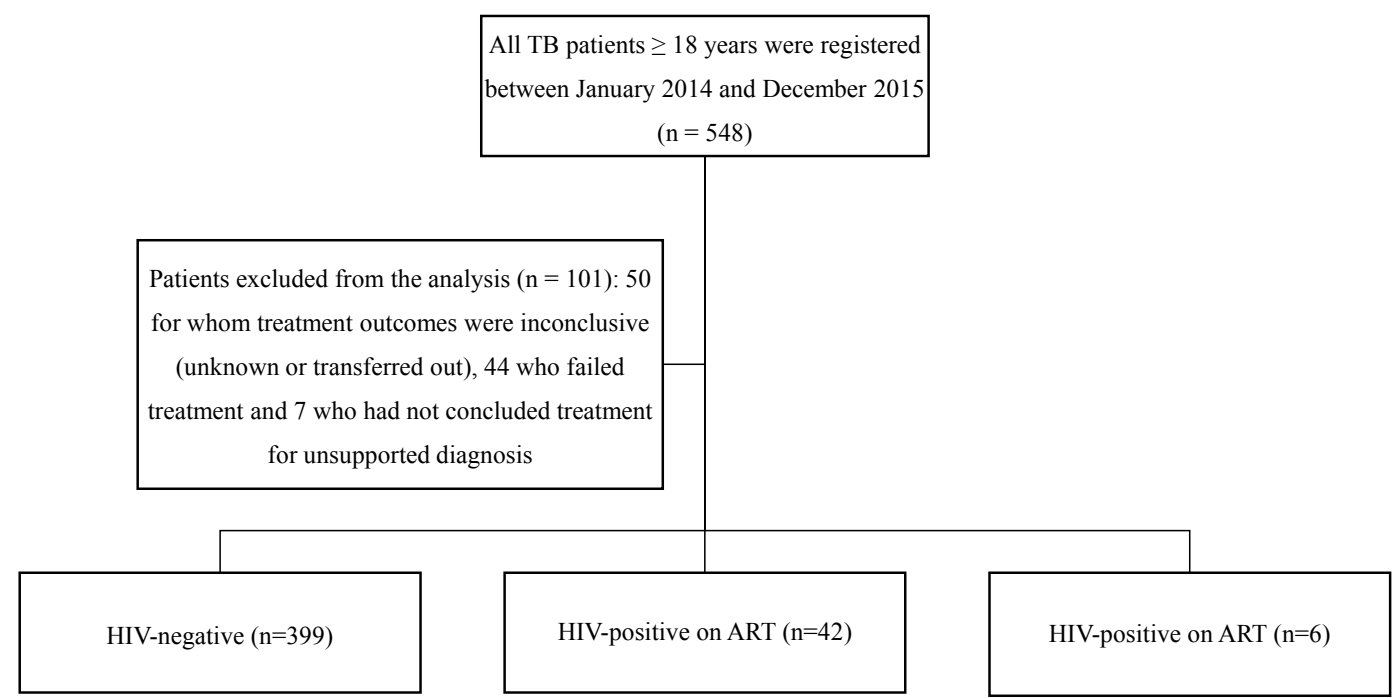

Figure 1. Flow diagram showing the HIV status and uptake of antiretroviral treatment for new TB patients $\geq 18$ years who were registered between January 2014 and December 2015 . 
meeting the inclusion criteria were obtained and included TB registration number, registration date, age, gender, place of residence, education, smoking behavior, TB presentation, HIV status, $\mathrm{CD} 4$ count at TB diagnosis, ART status, TB treatment outcome and TB treatment duration. Patient information was anonymized and de-identified preceding the analysis. Ethical approval for anonymized data collection was waived because of the retrospective nature of the study.

\subsection{Outcome Measures and Definitions}

We used internationally recommended definitions for TB treatment outcomes, namely cured, treatment completed and death [15]. Cure was defined as the occurrence of a negative sputum smear at the last month of treatment and at least on one additional occasion during the TB treatment. Treatment completion was defined as completed TB treatment, but for whom smear examination results were not complete to categorize the patient as cured; or based on clinical, radiological and complementary examination criteria in patients that did not produce sputum for a smear examination. Treatment success referred to patients "cured" and with "treatment completed". Death was considered all-cause mortality after TB diagnosis and previously to the end of TB treatment.

\subsection{Anti-Tuberculosis and Antiretroviral Treatment}

Standardized anti-tuberculosis treatment for new adult TB cases was a four-drug regimen of isoniazid, rifampicin, pyrazinamide and ethambutol during the two initial months ("intensive phase") followed by isoniazid and rifampicin during at least four months ("continuation phase") [16]. Antiretroviral therapy was accessible to HIV-positive patients not on ART according to the European and Portuguese ART guidelines [17] [18].

\subsection{Statistical Analysis}

Patient characteristics were organized by HIV and ART status (categorized as HIV-positive on ART, HIV-positive not on ART and HIV-negative). In order to compare categorical variables, chi-square test or Fisher's exact test were used. To compare the distribution of continuous variables, the Kruskal-Wallis test or Wilcoxon rank-sum were used. Multinomial logistic regression models were used to study the effects of HIV status, CD4 count and ART status on TB treatment outcome. In the multinomial logistic regression analysis, "treatment success" was the reference category for the dependent variable, being compared with the other category (death versus treatment success). Independent variables were as follows: age (continuous); gender (male or female); place of residence (rural or urban); education (none or any degree of education), type of TB (smear-negative pulmonary tuberculosis, smear-positive pulmonary tuberculosis or extra-pulmonary tuberculosis); CD4 count at the time of diagnosis $(<200$, 200 - 349, 350 - 499 or $\geq 500$ cells/ $\mu \mathrm{L}$ ); HIV status (HIV-positive on ART, HIV-positive not on ART or HIV-negative) and ART status (HIV-positive on 
ART or HIV-positive not on ART). All analyses were performed using statistical package of social sciences (SPSS) version 20.0 (SPSS Inc., Chicago, IL, USA) and the level of significance was p-value $<0.05 .95 \%$ confidence intervals were also calculated.

\section{Results}

A total of 548 new TB patients aged $\geq 18$ years old were registered between January 2014 and December 2015 (Figure 1). The new TB patients $(n=101)$ for whom TB treatment completion status was inconclusive (unknown or transferred out) or who failed TB treatment were exclude (Figure 1). The patient cohort was then formed by 447 patients. HIV status in this sample was classified as HIV-negative, in 399 patients (89.3\%) and HIV-positive, in 48 patients (10.7\%). HIV-positive on ART, in 42 patients (9.4\%) and HIV-positive not on ART, in 6 patients (1.3\%) (Table 1$)$.

The proportion of male patients was higher in all groups $(\mathrm{p}=0.003$; Table 1$)$. The proportion of patients with smear-positive TB was higher among HIV-positive not on ART $(p=0.005)$. No significant differences were established in age, place of residence, education or smoking behavior for each category. The death rate was higher in HIV-positive not on ART compared to the other groups ( $\mathrm{p}<$ 0.001) (Table 1). Among HIV-positive TB patients $(n=48)$, the median CD4 count was higher in patients HIV-positive on ART than HIV-positive not on ART (Table 1). The proportion of patients who had extended TB therapy was higher among HIV-positive patients $(\mathrm{p}=0.03)$. Only 6 patients $(12.5 \%)$ of HIV-positive TB patients eligible for ART did not initiate ART during their TB treatment (Figure 1).

In Table 2 are presented the results of multinomial logistic regression analysis

Table 1. Patients characteristics by HIV status among 447 new TB patients $\geq 18$ years who were registered between January 2014 and December 2015.

\begin{tabular}{cccccc}
\hline & Total & HIV-negative & $\begin{array}{c}\text { HIV-positive } \\
\text { on ART } \\
\text { N (\%) }\end{array}$ & $\begin{array}{c}\text { HIV-positive } \\
\text { not on ART } \\
\text { N (\%) }\end{array}$ & p-value \\
\hline Age, mean \pm SD (range) & $\begin{array}{c}51.7 \pm 19.0 \\
(18-96)\end{array}$ & $\begin{array}{c}52.4 \pm 19.0 \\
(18-96)\end{array}$ & $\begin{array}{c}46.2 \pm 19.2 \\
(22-68)\end{array}$ & $\begin{array}{c}50.5 \pm 19.1 \\
(33-64)\end{array}$ & 0.20 \\
Gender & & & & & \\
Male & $271(60.6)$ & $231(57.9)$ & $35(83.3)$ & $5(83.3)$ & 0.003 \\
Female & $176(39.4)$ & $168(42.1)$ & $7(16.7)$ & $1(16.7)$ & \\
Place of residence & & & & & \\
Rural & $103(23.0)$ & $93(23.3)$ & $9(21.4)$ & $1(16.7)$ & 0.90 \\
Urban & $344(77.0)$ & $306(76.7)$ & $33(78.6)$ & $5(83.3)$ & \\
Education & & & & & \\
None & $13(2.9)$ & $10(2.5)$ & $2(4.8)$ & $1(16.7)$ & 0.09 \\
Any degree of education & $434(97.1)$ & $389(97.5)$ & $40(95.2)$ & $5(83.3)$ & \\
Smoking behaviour & & & & & \\
\hline
\end{tabular}




\section{Continued}

\begin{tabular}{|c|c|c|c|c|c|}
\hline Non-smoker & $218(48.8)$ & $203(50.9)$ & $13(31.0)$ & $2(33.3)$ & \multirow[t]{3}{*}{0.10} \\
\hline Previous smoker & $167(37.4)$ & $145(36.3)$ & $19(45.2)$ & $3(50.0)$ & \\
\hline Active smoker & $62(13.9)$ & $51(12.8)$ & $10(23.8)$ & $1(16.7)$ & \\
\hline \multicolumn{6}{|l|}{ Type of TB } \\
\hline Smear-positive TB & $76(17.0)$ & $62(15.5)$ & $10(23.8)$ & $4(66.7)$ & \multirow{3}{*}{0.005} \\
\hline Smear-negative TB & $217(48.5)$ & $200(50.1)$ & $15(35.7)$ & $2(33.3)$ & \\
\hline ЕРТВ & $154(34.5)$ & $137(34.3)$ & $17(40.5)$ & 0 & \\
\hline \multicolumn{6}{|l|}{ CD4 count, cells/ $\mu \mathrm{L}$} \\
\hline Mean \pm SD (range) & $\begin{array}{c}283.35 \pm 176.68 \\
\quad(11-741)\end{array}$ & - & $\begin{array}{c}308.12 \pm 178.85 \\
(11-741)\end{array}$ & $\begin{array}{c}118.23 \pm 184.76 \\
(49-464)\end{array}$ & 0.9 \\
\hline$<200$ & $22(45.8)$ & - & $17(40.5)$ & $5(83.3)$ & \multirow{4}{*}{0.17} \\
\hline $200-349$ & $11(22.9)$ & - & $11(26.2)$ & - & \\
\hline $350-499$ & $6(12.5)$ & - & $5(11.9)$ & $1(16.7)$ & \\
\hline$\geq 500$ & $9(18.9)$ & - & $9(21.4)$ & - & \\
\hline \multicolumn{6}{|l|}{ TB treatment outcome } \\
\hline Sucess $^{\mathrm{a}}$ & $439(98.2)$ & $397(99.5)$ & $40(95.2)$ & $2(33.3)$ & \multirow{2}{*}{$<0.001$} \\
\hline Died & $8(1.8)$ & $2(0.5)$ & $2(4.8)$ & $4(66.7)$ & \\
\hline \multicolumn{6}{|l|}{ TB treatment duration } \\
\hline Prolonged treatment & $45(10.1)$ & $35(8.8)$ & $9(21.4)$ & $1(16.7)$ & \multirow{2}{*}{0.03} \\
\hline Standard treatment & $402(89.9)$ & $364(91.2)$ & $33(78.6)$ & $5(83.3)$ & \\
\hline Total & $447(100)$ & $399(89.3)$ & $42(9.4)$ & $6(1.3)$ & - \\
\hline
\end{tabular}

EPTB extra-pulmonary tuberculosis; Percentages may not add up to 100 due to rounding; ${ }^{a}$ Defined as cured and treatment completed.

Table 2. Multinomial logistic regression of factors associated with treatment outcomes in new TB patients.

\begin{tabular}{|c|c|c|}
\hline & $\begin{array}{c}\text { Died vs. Success } \\
\text { Unadjusted OR }(95 \% \mathrm{CI})\end{array}$ & $\begin{array}{c}\text { Died vs. Success } \\
\text { Adjusted OR }(95 \% \mathrm{CI})^{\mathrm{a}}\end{array}$ \\
\hline Age, years & $1.02(0.99$ to 1.06$)$ & $1.08(1.01 \text { to } 1.17)^{* * *}$ \\
\hline \multicolumn{3}{|l|}{ Gender } \\
\hline Male & Ref. & Ref. \\
\hline Female & $0.22(0.03$ to 1.77$)$ & $1.80(0.18$ to 18.30$)$ \\
\hline \multicolumn{3}{|l|}{ Type of TB } \\
\hline ЕРТВ & Ref. & Ref. \\
\hline Smear-negative TB & $3.61(0.42$ to 31.20$)$ & $3.65(0.37$ to 35.90$)$ \\
\hline Smear-positive TB & $4.14(0.37$ to 46.34$)$ & $0.57(0.003$ to 84.11$)$ \\
\hline \multicolumn{3}{|l|}{ HIV status } \\
\hline HIV-negative & Ref. & Ref. \\
\hline HIV-positive on ART & $9.93(1.36 \text { to } 72.37)^{\star}$ & $22.55(1.54 \text { to } 330.40)^{* * *}$ \\
\hline HIV-positive not on ART & $397.00(44.27 \text { to } 3559.91)^{* *}$ & $15,393.83(46.26 \text { to } 5,122,479.70)^{* * * *}$ \\
\hline
\end{tabular}


of the factors associated with treatment outcomes in new TB patients. The odds ratio (OR) for death in relation to treatment success was found to be significantly higher in patients HIV positive on ART (unadjusted OR 9.93; 95\% confidence interval (CI) 1.36 - 72.37) and HIV positive not on ART (unadjusted OR 397.00; 95\% confidence interval (CI) 44.27 to 3559.91 ) than in those HIV-negative. In the multinomial logistic regression analysis adjusting for age, gender, type of TB, and the effects of HIV status on the outcome of treatment were almost similar to those observed in the unadjusted multinomial logistic regression. Increased age was additionally associated with higher death rates regarding treatment success (adjusted OR 1.08; 95\% CI 1.01 - 1.17).

The results of the multinomial regression analysis of the factors associated with treatment outcomes in HIV-positive TB patients showed that the OR for death concerning to treatment success was higher in patients HIV-positive not on ART (unadjusted OR 40.00; 95\% CI 4.37 to 365.77) than in HIV-positive patients on ART. In the multinomial logistic regression analysis adjusting for age, gender, type of $\mathrm{TB}, \mathrm{CD}^{+}$cell count, and the effects of HIV positive status on $\mathrm{ART}$ and not on ART on the outcome of treatment were comparable to those observed in the unadjusted multinomial logistic regression.

\section{Discussion}

This study evaluated the impact of HIV, CD4 cell count and ART status on TB treatment outcomes in 447 new TB patients. Death rates were considerably higher in HIV-positive patients on ART and HIV-positive patients not on ART than in HIV-negative patients. Our study has some conflicting results when compared to some data from high-burden TB/HIV countries. A study among patients accessing an integrated TB and ART service in South Africa showed no differences in death rates between HIV-positive TB patients on ART and HIV-negative TB patients, but similar results to ours when comparing HIV-positive patients not on ART to HIV-negative patients concerning death rates [19]. This discrepancy is probable connected to the dissimilar prevalence of other diseases between different populations, differences in institutional tasks and patient's selection criteria. ART during a TB treatment event significantly improve mortality rates for the HIV-positive TB patients. Our data evidently designate that ART uptake should be upgraded with an improved follow-up of patients with HIV-associated TB. Our study result that throughout a TB treatment ART uptake was significantly associated with improved mortality rates, is comparable to studies with patients using acombined TB and ART service in high-burden countries [11] [12] [13]. In our study, we did not find an impact of CD4 cell counts on mortality. Several studies from Africa [20] [21] [22] [23], established that higher CD4 cell counts $(\geq 200$ cells $/ \mu \mathrm{L})$ were related with reduced mortality, suggesting that patients with CD4 counts $<200$ cells/ $\mu \mathrm{L}$ should be rapidly outlined for ART. The highlight of this study is that it provides an understanding of HIV, ART status and CD4 cell count impact on TB treatment outcomes among 
new TB patients in a low-burden country. The study findings, are useful to improve TB treatment outcomes among new patients with HIV-associated TB.

In this study, type of TB was not significantly associated with TB treatment outcomes in new TB patients and in the subgroup of HIV-positive TB patients. Previous studies from high-burden countries, revealed that comparing to smear-positive TB patients, the odds of mortality were 4 times higher for smear-negative patients and 2.7 times higher for extrapulmonary patients [24]. Additionally, current literature also showed a higher prevalence of HIV in patients with smear-negative pulmonary TB and extrapulmonary TB. TB diagnosis challenges and the resulting postponement in treatment initiation may result in higher mortality among smear-negative pulmonary $\mathrm{TB}$ and extrapulmonary $\mathrm{TB}$ patients [25].

Despite medical progress, numerous key challenges to TB-HIV co-infection control remain, as this potentially deadly combination remains relevant in Portugal and WHO European Region. Improved development of collaborative structures and mechanisms for the integrated TB-HIV care may facilitate the delivery of treatment to TB-HIV patients. Additionally, all HIV patients should have access to integrated, patient-centred and preventive TB strategies and supporting systems. Increased research and innovation is also needed in this area.

Most of the evidence supporting these findings has arisen from retrospective and single-center studies. Our study has several potential limitations. First, our analysis was retrospective in nature. Second, we evaluated patients treated at a single institution, not reflecting approaches at other medical centers and was carried out in a dedicated operational unit, with the cooperation of skilled personnel. Additionally, we used only routinely collected data that without detailed patients HIV-RNA viral loads and antiretroviral treatment histories of patients already on ART at the time of TB diagnosis (clinical immunological or virological treatment failure and antiretroviral resistance). Consequently, the study could not search these factors impact on TB treatment outcomes. A prospective and multicentric study is required to validate our results.

\section{Conclusion}

In conclusion, this study showed that there was significant difference in death rates between HIV-positive and HIV-negative TB patients. HIV-positive patients not on ART had a significantly higher mortality. These findings strain the need for an intervention aimed at preventing TB-HIV co-infection and improving ART uptake. Further research is needed into the burden and associated risk factors of co-infection in Europe, to help plan effective control measures. Increased HIV testing of TB patients and targeted and informed strategies for control and prevention could help curb the co-infection epidemic.

\section{Acknowledgements}

We are most grateful to all the staff members of the Pulmonary Diagnosis Cen- 
ter Dr. Ribeiro Sanches.

\section{Funding}

This study was not supported financially by any institutional/governmental and nongovernmental organization.

\section{Conflicts of Interest}

There is no conflicting interest as declared by the authors for this research.

\section{References}

[1] (2017) Global Tuberculosis Report. World Health Organization, Geneva.

[2] Sharma, S.K., Mohan, A. and Kadhiravan, T. (2005) HIV-TB Co-Infection: Epidemiology, Diagnosis and Management. Indian Journal of Medical Research, 121, 550-567.

[3] Diniz, A., Duarte, R., Bettencourt, J., Melo, T., Gomes, M. and Oliveira, O. (2015) Portugal, Infeçãopor VIH, SIDA e Tuberculoseemnúmeros, Pinto Azul, Unipessoal Lda, Lisboa.

[4] Pimpin, L., Drumright, L.N., Kruijshaar, M.E., Abubakar, I., Rice, B., Delpech, V., et al. (2011) Tuberculosis and HIV Co-Infection in European Union and European Economic Area Countries. European Respiratory Journal, 38, 1382-1392. https://doi.org/10.1183/09031936.00198410

[5] Kwan, C.K. and Ernst, J.D. (2011) HIV and Tuberculosis: A Deadly Human Syndemic. Clinical Microbiology Reviews, 24, 351-376.

https://doi.org/10.1128/CMR.00042-10

[6] Harries, A., Maher, D. and Graham, S. (2004) TB/HIV: A Clinical Manual. 2th Edition, World Health Organization.

[7] Banerjee, A., Moyo, S., Salaniponi, F. and Harries, A. (1997) HIV Testing and Tuberculosis Treatment Outcome in a Rural District in Malawi. Transactions of the Royal Society of Tropical Medicine and Hygiene, 91, 707-708. https://doi.org/10.1016/S0035-9203(97)90533-2

[8] Sume, G.E., Hoshen, M., Bita, G., Kabore, S. and Nzima, V.N. (2009) Treatment Outcome of TB/HIV Positive and Negative Smear Positive Pulmonary Tuberculosis Patients Treated Using Daily Self-Administered Therapy in a Cameroonian District Hospital. East African Medical Journal, 86, 469-475.

[9] Daniel, O.J. and Alausa, O.K. (2006) Treatment Outcome of TB/HIV Positive and TB/HIV Negative Patients on Directly Observed Treatment, Short Course (DOTS) in Sagamu, Nigeria. Nigerian Journal of Medicine, 15, 222-226.

[10] Shaweno, D. and Worku, A. (2012) Tuberculosis Treatment Survival of HIV Positive TB Patients on Directly Observed Treatment Short-Course in Southern Ethiopia: A Retrospective Cohort Study. BMC Research Notes, 5, 682. https://doi.org/10.1186/1756-0500-5-682

[11] Tweya, H., Feldacker, C., Phiri, S., Ben-Smith, A., Fenner, L., Jahn, A., et al. (2013) Comparison of Treatment Outcomes of New Smear-Positive Pulmonary Tuberculosis Patients by HIV and Antiretroviral Status in a TB/HIV Clinic, Malawi. PLoS One, 8, e56248. https://doi.org/10.1371/journal.pone.0056248

[12] Njepoume, N., Odume, B. and Suraj, A. (2009) The Impact of HIV Syndroms on the Treatment Outcome of TB Cases: Results from a State TB Programme in Nigeria. 
International Journal of Tuberculosis and Lung Disease, 13, 316.

[13] van den Broek, J., Mfinanga, S., Moshiro, C., O’Brien, R., Mugomela, A. and Lefi, M. (1998) Impact of Human Immunodeficiency Virus Infection on the Outcome of Treatment and Survival of Tuberculosis Patients in Mwanza, Tanzania. International Journal of Tuberculosis and Lung Disease, 2, 547-552.

[14] El-Sony, A.I., Khamis, A.H., Enarson, D.A., Baraka, O., Mustafa, S.A. and Bjune, G. (2002) Treatment Results of DOTS in 1797 Sudanese Tuberculosis Patients with or without HIV Co-Infection. International Journal of Tuberculosis and Lung Disease, 6, 1058-66.

[15] Fujiwara, P.I., Dlodlo, R.A., Ferroussier, O., Nakanwagi-Mukwaya A., Cesari, G. and Boillot, E. (2012) Implementing Collaborative TB-HIV Activities: A Programmatic Guide. Paris, France. International Union against Tuberculosis and Lung Disease.

[16] Yew, W.W., Lange, C. and Leung, C.C. (2011) Treatment of Tuberculosis: Update 2010. European Respiratory Journal, 37, 441-462.

https://doi.org/10.1183/09031936.00033010

[17] Panel on Antiretroviral Guidelines for Adults and Adolescents (2011) Guidelines for the Use of Antiretroviral Agents in HIV-1-Infected Adults and Adolescents. Department of Health and Human Services, 14 October 2011, 1-167.

[18] Guerreiro, C., Aldir, I., Oliveira, J., Mansinho, K., Marques, L., Doroana, M., et al. (2016) Recomendações Portuguesas para o tratamento da infeção por VIH-1 e VIH-2, Versão 1.0.

[19] Mweete, D., Nglazi1, Bekker L., Wood, R. and Kaplan R. (2015) The Impact of HIV Status and Antiretroviral Treatment on TB Treatment Outcomes of New Tuberculosis Patients Attending Collocated TB and ART Services in South Africa: A Retrospective Cohort Study. BMC Infectious Diseases, 15, 536. https://doi.org/10.1186/s12879-015-1275-3

[20] Lawn, S.D., Myer, L., Edwards, D., Bekker, L.G. and Wood, R. (2009) Short-Term and Long-Term Risk of Tuberculosis Associated with CD4 Cell Recovery during Antiretroviral Therapy in South Africa. AIDS, 23, 1717-1725. https://doi.org/10.1097/QAD.0b013e32832d3b6d

[21] Karim, S.S., Naidoo, K., Grobler, A., Padayatchi, N., Baxter, C., Gray, A.L., et al. (2011) Integration of Antiretroviral Therapy with Tuberculosis Treatment. The New England Journal of Medicine, 365, 1492-1501. https://doi.org/10.1056/NEJMoa1014181

[22] Blanc, F.X., Sok, T., Laureillard, D., Borand, L., Rekacewicz, C., Nerrienet, E., et al. (2011) Earlier versus Later Start of Antiretroviral Therapy in HIV-Infected Adults with Tuberculosis. The New England Journal of Medicine, 365, 1471-1481. https://doi.org/10.1056/NEJMoa1013911

[23] Havlir, D.V., Kendall, M.A., Ive, P., Kumwenda, J., Swindells, S., Qasba, S.S., et al. (2011) Timing of Antiretroviral Therapy for HIV-1 Infection and Tuberculosis. The New England Journal of Medicine, 365, 1482-1491. https://doi.org/10.1056/NEJMoa1013607

[24] Gebremariam, G., Asmamaw, G., Hussen, M., Hailemariam, M.Z., Asegu, D., Astatkie, A., et al. (2016) Impact of HIV Status on Treatment Outcome of Tuberculosis Patients Registered at Arsi Negele Health Center, Southern Ethiopia: A Six Year Retrospective Study. PLoS ONE, 11, e0153239. https://doi.org/10.1371/journal.pone.0153239

[25] Sanchez, M., Bartholomay, P., Arakaki-Sanchez, D., Enarson, D., Bissell, K., et al. 
(2012) Outcomes of TB Treatment by HIV Status in National Recording Systems in Brazil, 2003-2008. PLOS ONE, 7, e33129.

https://doi.org/10.1371/journal.pone.0033129 